PROCEEDINGS OF THE

AMERICAN MATHEMATICAL SOCIETY

Volume 131, Number 9, Pages 2953-2962

S 0002-9939(03)06847-3

Article electronically published on January 28, 2003

\title{
PROOFS OF TWO CONJECTURES OF GRAY INVOLVING THE DOUBLE SUSPENSION
}

\author{
STEPHEN D. THERIAULT \\ (Communicated by Paul Goerss)
}

\begin{abstract}
In proving that the fiber of the double suspension has a classifying space, Gray constructed fibrations

$$
S^{2 n-1} \stackrel{E^{2}}{\longrightarrow} \Omega^{2} S^{2 n+1} \stackrel{f}{\rightarrow} B W_{n}
$$

and

$$
B W_{n} \rightarrow \Omega S^{2 n p+1} \stackrel{\phi}{\longrightarrow} S^{2 n p-1} .
$$

He conjectured that $E^{2} \circ \phi$ is homotopic to the $p^{t h}$-power map on $\Omega^{2} S^{2 n p+1}$ when $p$ is an odd prime. Harper proved this is true when looped once. We remove the loop when $p \geq 5$. Gray also conjectured that at odd primes $f$ factors through a map

$$
\Omega S^{2 n+1}\{p\} \rightarrow B W_{n} .
$$
\end{abstract}

We show that this is true as well when $p \geq 5$.

\section{INTRODUCTION}

The purpose of this paper is to positively resolve two conjectures of Gray. Both concern statements at odd primes so we will assume from the outset that all spaces and maps have been localized at an odd prime $p$. In [G1] Gray constructs a classifying space for the fiber of the double suspension

$$
W_{n} \longrightarrow S^{2 n-1} \stackrel{E^{2}}{\longrightarrow} \Omega^{2} S^{2 n+1} .
$$

In particular, he produces homotopy fibrations

$$
S^{2 n-1} \stackrel{E^{2}}{\longrightarrow} \Omega^{2} S^{2 n+1} \stackrel{f}{\longrightarrow} B W_{n}
$$

and

$$
B W_{n} \stackrel{g}{\longrightarrow} \Omega^{2} S^{2 n p+1} \stackrel{\phi}{\longrightarrow} S^{2 n p-1}
$$

with the properties that: (1) $g \circ f \simeq \Omega H$, where

$$
H: \Omega S^{2 n+1} \longrightarrow \Omega S^{2 n p+1}
$$

is the $p^{t h}$ James-Hopf invariant, (2) $\Omega \phi$ factors as the composite

$$
\Omega^{3} S^{2 n p+1} \stackrel{\Omega P}{\longrightarrow} \Omega J_{p-1}\left(S^{2 n}\right) \stackrel{T}{\longrightarrow} \Omega S^{2 n p-1},
$$

Received by the editors September 28, 2001 and, in revised form, April 2, 2002.

2000 Mathematics Subject Classification. Primary 55P40; Secondary 55R35.

Key words and phrases. $p^{\text {th }}$-power map, double suspension. 
where $P$ is the connecting map in the $E H P$ sequence, and $T$ is the Toda map, and (3) $f$ and $g$ can be chosen to be $H$-maps if $p \geq 5$.

This setup also leads to a factorization of the $p^{t h}$-power map on $\Omega^{3} S^{2 n p+1}$. In particular, the composite

$$
B W_{n} \stackrel{g}{\longrightarrow} \Omega^{2} S^{2 n p+1} \stackrel{p}{\longrightarrow} \Omega^{2} S^{2 n p+1}
$$

is null homotopic, leading to a homotopy commutative square

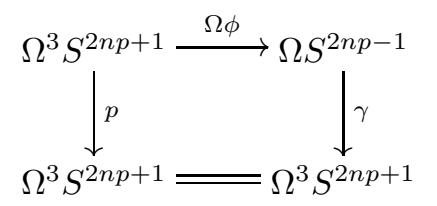

for some map $\gamma$. In $[\underline{\mathrm{H}}$, Harper filled in the missing piece by showing that $\gamma$ could be chosen to be $\Omega E^{2}$. Gray has conjectured that this factorization should deloop. We prove that it does, given a suitable choice of the map $\phi$.

Theorem 1.1. Let $p \geq 5$. Then there is a map

$$
\Omega^{2} S^{2 n p+1} \stackrel{\phi^{\prime}}{\longrightarrow} S^{2 n p-1}
$$

such that

$$
B W_{n} \stackrel{g}{\longrightarrow} \Omega^{2} S^{2 n p+1} \stackrel{\phi^{\prime}}{\longrightarrow} S^{2 n p-1}
$$

is a homotopy fibration and $E^{2} \circ \phi^{\prime} \simeq p$.

The restriction to primes larger than 3 comes from our use of the multiplicativity of the maps $f$ and $g$.

The factorization in Theorem 1.1 ties into work of Cohen, Moore, and Neisendorfer CMN. Using different methods, they construct a map

$$
\pi_{2 n}: \Omega^{2} S^{2 n+1} \longrightarrow S^{2 n-1}
$$

such that $E^{2} \circ \pi_{2 n} \simeq p$. This was given its optimal formulation by Anick [A], who constructed a homotopy fibration sequence

$$
\Omega^{2} S^{2 n+1} \stackrel{\pi_{2 n}}{\longrightarrow} S^{2 n-1} \longrightarrow T^{2 n-1} \longrightarrow \Omega S^{2 n+1} .
$$

In particular, if $\phi$ and $\pi_{2 n p}$ were homotopic (or homotopic when looped), then $W_{n}$ has $T^{2 n p-1}$ as a double classifying space.

The second of Gray's conjectures can be considered a first test of whether his approach meshes with that of Cohen, Moore, and Neisendorfer. The map

$$
T^{2 n-1} \longrightarrow \Omega S^{2 n+1}
$$

lifts to $\Omega S^{2 n+1}\{p\}$, the fiber of the $p^{t h}$-power map on $\Omega S^{2 n+1}$. It follows that there is a homotopy pullback diagram:

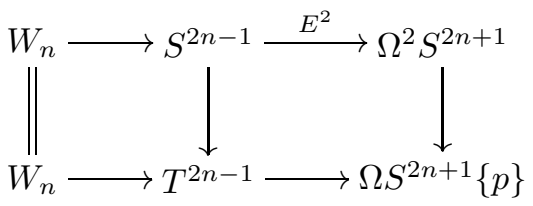

Gray's work proved that the upper horizontal fibration can be moved one step to the right with the map

$$
\Omega^{2} S^{2 n+1} \stackrel{f}{\longrightarrow} B W_{n} .
$$


The conjecture is that the lower horizontal fibration can also be moved one step to the right. Theorem 1.2 proves this is the case.

Theorem 1.2. If $p \geq 5$, there is a homotopy pullback diagram:

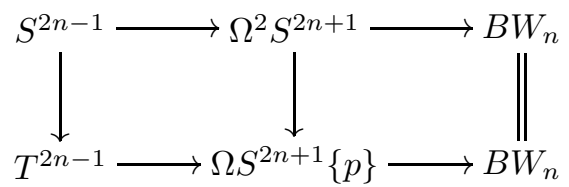

Theorem 1.2 also fits nicely into the program of [G2], which inductively constructs EHP-type sequences informing on the homotopy of Smith-Toda complexes. In this context, the fibration sequence from Theorem 1.2 is thought of as

$$
W_{n} \stackrel{P}{\longrightarrow} T^{2 n-1} \stackrel{E}{\longrightarrow} \Omega S^{2 n+1}\{p\} \stackrel{H}{\longrightarrow} B W_{n} .
$$

The techniques used in this paper should have further application. Both Theorems 1.1 and 1.2 are concerned with delooping some statement. At heart, this is done by using the multiplicativity of certain maps together with (essentially) the Hopf construction to produce advantageous null homotopies.

\section{TheOREM 1.1: THE FACTORIZATION OF THE $p^{t h}$-POWER MAP ON $\Omega^{2} S^{2 n p+1}$}

The two main ingredients in the proof of Theorem 1.1 are Lemmas 2.2] and 2.3.

It is a standard fact that for odd primes, the $p^{t h}$ James-Hopf invariant has order $p$ when looped. Selick $[\underline{\mathrm{S}}]$ proved the more subtle fact that the lift of $\Omega H$ to the fiber of the $p^{t h}$-power map on $\Omega^{2} S^{2 n p+1}$ can be chosen to be an $H$-map.

Theorem 2.1. If $p \geq 3$, then there is a homotopy commutative diagram

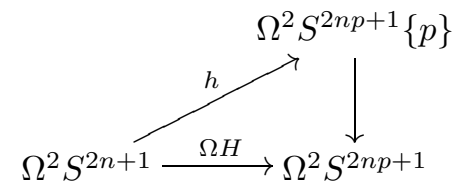

where $h$ is an H-map.

The next lemma says that the $H$-lift in Theorem 2.1 can be factored by $H$-maps through $B W_{n}$.

Lemma 2.2. If $p \geq 5$, there is a homotopy commutative diagram

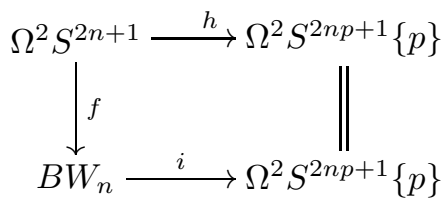

where all maps are $H$-maps.

Proof. Start with the $H$-maps $f$ and $h$. We first show that the asserted homotopy commutative diagram exists for some map $i$, and then show that such an $i$ must be an $H$-map. 
By [G1], there is a homotopy equivalence $\Sigma^{2} \Omega^{2} S^{2 n+1} \simeq \Sigma^{2}\left(S^{2 n-1} \times B W_{n}\right)$. In particular, $\Sigma^{2} f$ has a right homotopy inverse. Knowing this, the homotopy equivalence can be recast as the sum of three maps, the inclusion

$$
\Sigma^{2} S^{2 n-1} \stackrel{\Sigma^{2} E^{2}}{\longrightarrow} \Sigma^{2} \Omega^{2} S^{2 n+1}
$$

a right homotopy inverse

$$
s: \Sigma^{2} B W_{n} \longrightarrow \Sigma^{2} \Omega^{2} S^{2 n+1}
$$

of $f$, and a product term

$$
t: \Sigma^{2} B W_{n} \wedge S^{2 n-1} \stackrel{s \wedge E^{2}}{\longrightarrow} \Sigma^{2} \Omega^{2} S^{2 n+1} \wedge \Omega^{2} S^{2 n+1} \stackrel{\Sigma \mu}{\longrightarrow} \Sigma^{2} \Omega^{2} S^{2 n+1},
$$

where $\mu$ is the Hopf construction. The inclusion $E^{2}$ composes trivially with $h$ by connectivity, and so the multiplicativity of $h$ implies that $t$ also composes trivially with $\Sigma^{2} h$. Hence $\Sigma^{2} h$ factors through $\Sigma^{2} f$, giving a homotopy commutative diagram

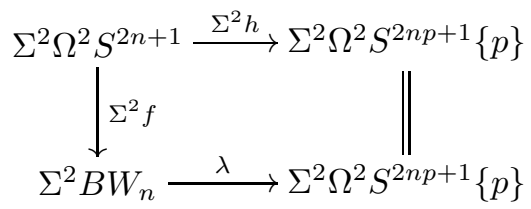

for some map $\lambda$. Now first evaluating

$$
\Sigma^{2} \Omega^{2} S^{2 n p+1}\{p\} \longrightarrow S^{2 n p+1}\{p\}
$$

and then taking adjoints gives the asserted factorization of $h$ for some map $i$.

To show that such an $i$ is an $H$-map, consider the following diagram:

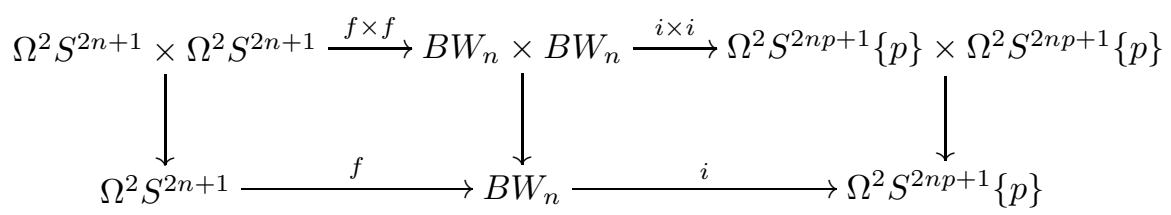

The outer and left squares homotopy commute. Since $\Sigma^{2}(f \times f)$ has a right homotopy inverse, the right square also homotopy commutes. Thus $i$ is an $H$-map.

The following lemma starts with a fibration over a suspension and identifies the cofiber of the fiber mapping into the total space.

Lemma 2.3. Suppose

$$
F \longrightarrow E \longrightarrow \Sigma B
$$

is a homotopy fibration. Then there is a homotopy cofibration sequence

$$
F \longrightarrow E \longrightarrow \Sigma B \vee(\Sigma B \wedge F) \stackrel{a \perp b}{\longrightarrow} \Sigma F,
$$

where $b$ is the map obtained from suspending the homotopy action

$$
\Omega \Sigma B \times F \longrightarrow F \text {. }
$$

Specifically, $b$ is the composite

$$
\Sigma B \wedge F \longrightarrow \Sigma \Omega \Sigma B \wedge F \longrightarrow \Sigma(\Omega \Sigma B \times F) \longrightarrow \Sigma F .
$$

Proof. This is a corollary of [G1, Prop. 1(a)]. 
Proof of Theorem 1.1. Consider the homotopy pullback of $H$-spaces and $H$-maps:

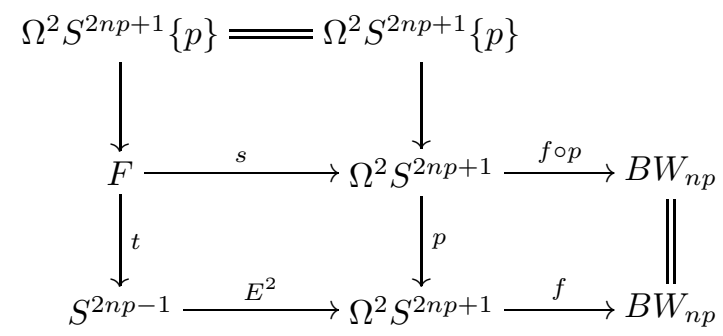

By [CMN], the $p^{t h}$-power map on $\Omega^{2} S^{2 n p+1}$ factors through the double suspension. Hence $f \circ p \simeq *$ and so $F \simeq \Omega^{2} S^{2 n p+1} \times W_{n}$. What is at issue, though, is the choice of splitting. We wish to show that $s$ has a right homotopy inverse which gives a factorization of the $p^{\text {th }}$-power map through the double suspension and which, when composed with $t$, has fiber $B W_{n}$. That is the statement in Theorem 1.1.

Applying Lemma 2.3 to the homotopy fibration

$$
B W_{n} \stackrel{g}{\longrightarrow} \Omega^{2} S^{2 n p+1} \stackrel{\phi}{\longrightarrow} S^{2 n p-1}
$$

we obtain a homotopy cofibration sequence

$$
B W_{n} \stackrel{g}{\longrightarrow} \Omega^{2} S^{2 n p+1} \longrightarrow S^{2 n p-1} \vee\left(S^{2 n p-1} \wedge B W_{n}\right) \stackrel{a \perp b}{\longrightarrow} \Sigma B W_{n} .
$$

Using the $H$-map $i$ in Lemma 2.2, let $\theta$ be the composite of $H$-maps

$$
\theta: B W_{n} \stackrel{i}{\longrightarrow} \Omega^{2} S^{2 n p+1}\{p\} \longrightarrow F .
$$

Since $F$ is $(2 n p-2)$-connected, the restriction of $\theta$ to the bottom cell of $B W_{n}$ is null homotopic. As $\theta$ is multiplicative, the definition of $b$ in Theorem 2.3 implies that the composite

$$
S^{2 n p-1} \wedge B W_{n} \stackrel{b}{\longrightarrow} \Sigma B W_{n} \stackrel{\Sigma \theta}{\longrightarrow} \Sigma F
$$

is also null homotopic. Hence

$$
S^{2 n p-1} \vee\left(S^{2 n p-1} \wedge B W_{n}\right) \stackrel{a \perp b}{\longrightarrow} \Sigma B W_{n} \stackrel{\Sigma \theta}{\longrightarrow} \Sigma F
$$

is null homotopic. Extending along the cofiber of $a \perp b$ we obtain a homotopy commutative diagram

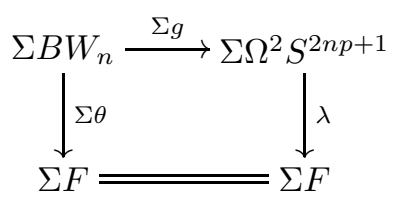

for some map $\lambda$. Looping this diagram, using the natural inclusion

$$
X \stackrel{E}{\longrightarrow} \Omega \Sigma X
$$

and using the fact that $F$ is an $H$-space so there is a retraction

$$
\Omega \Sigma F \stackrel{r}{\longrightarrow} F
$$


with $r \circ E \simeq 1_{F}$, we obtain a homotopy commutative diagram

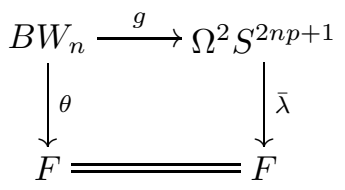

where $\bar{\lambda}=r \circ \Omega \lambda \circ E$.

First consider what happens when we compose $\bar{\lambda}$ with

$$
F \stackrel{t}{\longrightarrow} S^{2 n p-1} \text {. }
$$

As $\theta$ was defined to factor through the fiber of $t$, we have $t \circ \bar{\lambda} \circ g \simeq *$.

Next consider what happens when we compose $\bar{\lambda}$ with

$$
F \stackrel{s}{\longrightarrow} \Omega^{2} S^{2 n p+1} \text {. }
$$

The pullback defining $F$ and the definition of $\theta$ imply that $s \circ \theta=g$. Hence we have a homotopy commutative diagram

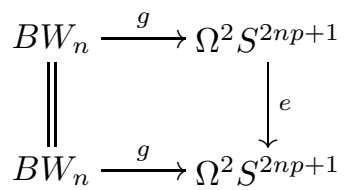

where $e=s \circ \bar{\lambda}$. In particular, $e$ is a homology isomorphism and so is a homotopy equivalence. This gives: (1) a splitting $F \simeq \Omega^{2} S^{2 n p+1} \times W_{n}$, and (2) from the pullback defining $F$, a homotopy $E^{2} \circ t \circ \bar{\lambda} \simeq p \circ e$. Now, from the previous paragraph, $B W_{n}$ lifts to the fiber of $t \circ \bar{\lambda}$, and the homology information from the homotopy in (2) tells us that the lift is a homotopy equivalence. Thus there is a homotopy fibration

$$
B W_{n} \stackrel{g}{\longrightarrow} \Omega^{2} S^{2 n p+1} \stackrel{t \circ \bar{\lambda}}{\longrightarrow} S^{2 n p-1} .
$$

To complete the proof of Theorem 1.1 we have to remove the homotopy equivalence $e$. Define $\phi^{\prime}$ as the composite

$$
\phi^{\prime}: \Omega^{2} S^{2 n p+1} \stackrel{e^{-1}}{\longrightarrow} \Omega^{2} S^{2 n p+1} \stackrel{\bar{\lambda}}{\longrightarrow} F \longrightarrow S^{2 n p-1} .
$$

Then $E^{2} \circ \phi^{\prime} \simeq p$. Also, our care in choosing $\bar{\lambda}$ as a right homotopy inverse to

$$
F \stackrel{s}{\longrightarrow} \Omega^{2} S^{2 n p+1}
$$

now pays off. Since $e \circ g \simeq g$, we have $e^{-1} \circ g \simeq g$, and so there is a homotopy fibration

$$
B W_{n} \stackrel{g}{\longrightarrow} \Omega^{2} S^{2 n p+1} \stackrel{\phi^{\prime}}{\longrightarrow} S^{2 n p-1} .
$$

\section{Theorem 1.2: The Fibration $T^{2 n-1} \rightarrow \Omega S^{2 n p+1}\{p\} \rightarrow B W_{n}$}

Let $T=T^{2 n-1}$. Anick's space $T$ is an $H$-space so we can consider the homotopy pullback diagram

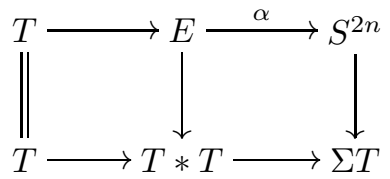


where the bottom row is the Hopf construction and the right vertical arrow is the inclusion of the bottom cell.

We begin by recording some facts regarding $E$. Let

$$
[\iota, \iota]: S^{4 n-1} \longrightarrow S^{2 n}
$$

be the Whitehead product of the identity map on $S^{2 n}$ with itself.

Lemma 3.1. The Whitehead product

$$
S^{4 n-1} \stackrel{[\iota, \iota]}{\longrightarrow} S^{2 n}
$$

factors through $\alpha$.

Proof. The pullback defining $E$ factors through the pullback

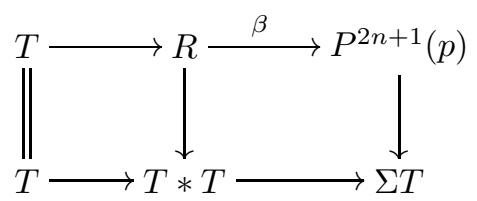

where $P^{2 n+1}(p)$ is the bottom Moore space including into $\Sigma T$. By [CMN] or [A], $R=P^{4 n}(p) \cup$ higher, and the restriction of $\beta$ to $P^{4 n}(p)$ is the composite

$$
P^{4 n}(p) \stackrel{[\nu, \mu]}{\longrightarrow} P^{2 n+1}(p) \longrightarrow G,
$$

where $[\nu, \mu]$ is the mod- $p$ Whitehead product of the identity $\nu$ and the Bockstein $\mu$ on $P^{2 n+1}(p)$. The lemma now follows once we observe that the restriction of $[\nu, \mu]$ to $S^{4 n-1}$ factors through $S^{2 n}$ via $[\iota, \iota]$.

Corollary 3.2. $\Omega E \simeq \Omega S^{4 n-1} \times \Omega^{2} S^{2 n+1}$.

Proof. The factorization in Lemma 3.1 implies that $\Omega S^{4 n-1}$ is a retract of $\Omega E$ because it is a retract of $\Omega S^{2 n}$. So it remains to identify the homotopy fiber of

$$
S^{4 n-1} \longrightarrow E \text {. }
$$

That this is $\Omega^{2} S^{2 n+1}$ follows from the homotopy pullback:

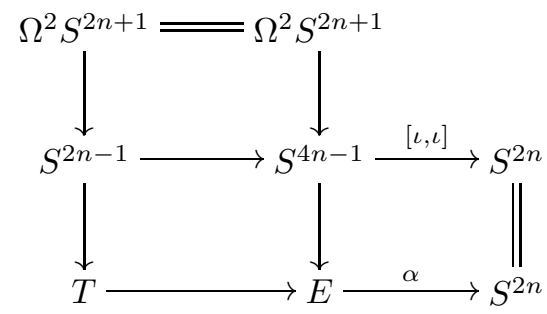

Lemma 3.3. There is a homotopy commutative diagram

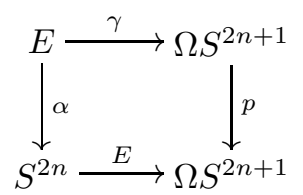

for some map $\gamma$ which is degree one in $H_{2 n}()$. 
Proof. By [T] the map

$$
T \longrightarrow \Omega S^{2 n+1}
$$

in Anick's fibration lifts to a map

$$
\delta: T \longrightarrow \Omega S^{2 n+1}\{p\}
$$

which can be chosen to be an $H$-map. Let $\tilde{\delta}$ be the adjoint of $\delta$. The naturality of the Hopf construction then implies that the composition

$$
T * T \longrightarrow \Sigma T \stackrel{\tilde{\delta}}{\longrightarrow} S^{2 n+1}\{p\}
$$

is null homotopic. Hence

$$
\psi: E \stackrel{\alpha}{\longrightarrow} S^{2 n} \longrightarrow \Sigma T \stackrel{\tilde{\delta}}{\longrightarrow} S^{2 n+1}\{p\}
$$

is null homotopic.

By connectivity there is a homotopy commutative diagram:

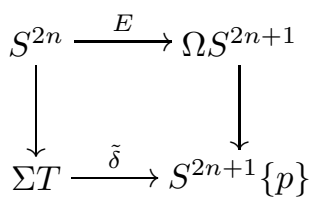

The null homotopy $\psi$ implies $E \circ g$ lifts through the $p^{t h}$ power map on $\Omega S^{2 n+1}$. Let $\gamma$ be some such lift. Then $\gamma$ is degree one in $H_{2 n}()$ because $\alpha$ is.

We now wish to examine the homotopy fiber of $\gamma$.

Lemma 3.4. There is a homotopy fibration

$$
S^{4 n-1} \longrightarrow E \stackrel{\gamma}{\longrightarrow} \Omega S^{2 n+1} .
$$

Further, the composite

$$
\theta: S^{4 n-1} \longrightarrow E \stackrel{\alpha}{\longrightarrow} S^{2 n}
$$

is homotopic to $[\iota, \iota]+\epsilon$, where $\epsilon \in \pi_{4 n-1}\left(S^{2 n}\right)$ is p-torsion.

Remark. It is likely that $\epsilon \simeq *$, but what is really important is the appearance of the Whitehead product $[\iota, \iota]$ in the homotopy for $\theta$.

Proof. Consider the homotopy pullback:

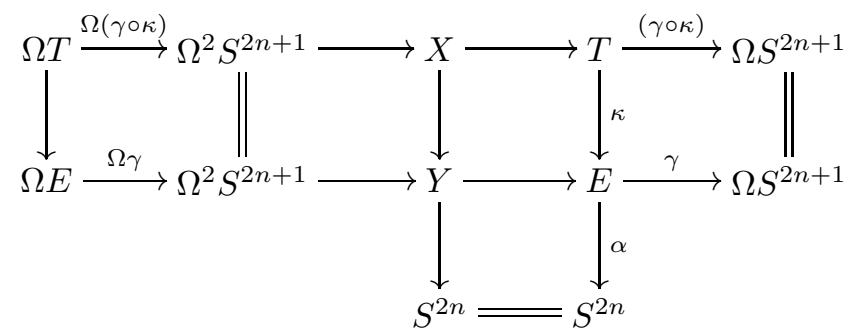

We first show that $X \simeq S^{2 n-1}$ and then use this to show that $Y \simeq S^{4 n-1}$. The latter homotopy equivalence proves the lemma. 
By connectivity, the inclusion of $S^{2 n-1}$ into $T$ factors through $X$. This gives a homotopy fibration diagram

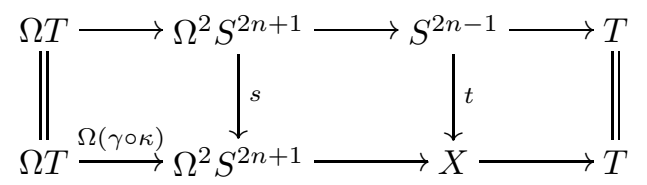

for some maps $s$ and $t$. By Lemma 3.3 the map $\Omega(\gamma \circ \kappa)$ is degree one in $H_{2 n-1}()$. Thus $s$ is degree one in $H_{2 n-1}()$. Hence $s$ is a homotopy equivalence since $\Omega^{2} S^{2 n+1}$ is atomic. This then implies that $t$ is a homotopy equivalence by the five-lemma.

Replacing $X$ by $S^{2 n-1}$, now consider the homotopy fibration

$$
S^{2 n-1} \longrightarrow Y \longrightarrow S^{2 n} \text {. }
$$

Again, since $\gamma$ is degree one in $H_{2 n}(), Y$ is $(4 n-2)$-connected. Rationally then, we have $Y \simeq S^{4 n-1}$. This implies that $p$-locally, when the bottom cell is included into $Y$, the composite

$$
\theta: S^{4 n-1} \longrightarrow Y \longrightarrow S^{2 n}
$$

is homotopic to $[\iota, \iota]+\epsilon$, where $\epsilon \in \pi_{4 n-1}\left(S^{2 n}\right)$ is $p$-torsion. The perturbation of the Whitehead product $[\iota, \iota]$ by $\epsilon$ does not affect the Serre spectral sequence calculation of the homology of the fiber of $\theta$. Thus the homotopy fiber of $\theta$ is homotopy equivalent to $S^{2 n+1}$. This gives a homotopy pullback diagram:

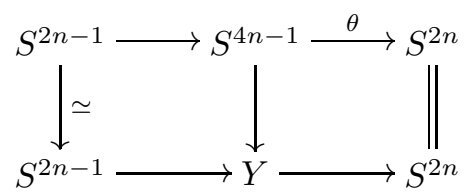

Hence $Y \simeq S^{4 n-1}$ by the five-lemma.

Proof of Theorem 1.2. It suffices to prove the existence of a homotopy fibration

$$
T^{2 n-1} \longrightarrow \Omega S^{2 n+1}\{p\} \longrightarrow B W_{n}
$$

a homology calculation then immediately implies that the homotopy fiber of the composite

$$
\Omega^{2} S^{2 n+1} \longrightarrow \Omega S^{2 n+1}\{p\} \longrightarrow B W_{n}
$$

is homotopy equivalent to $S^{2 n-1}$.

By [G1, Prop 4(c)], when $p \geq 3$, there is a homotopy fibration

$$
B W_{n} \times S^{4 n-1} \longrightarrow S^{2 n} \stackrel{E}{\longrightarrow} \Omega S^{2 n+1},
$$

where the restriction to $S^{4 n-1}$ in the left-hand map is the Whitehead product $[\iota, \iota]$. Consider the homotopy pullback diagram

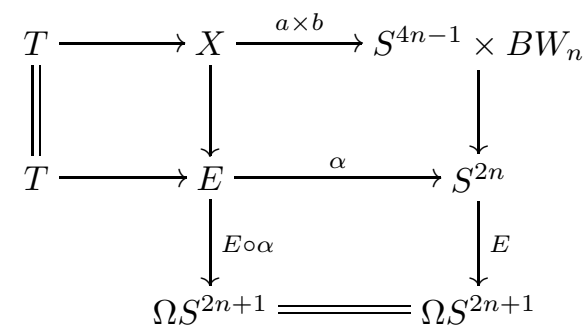


which defines the maps $a$ and $b$. Theorem 1.2 will follow if we can show that $X \simeq S^{4 n-1} \times \Omega S^{2 n+1}\{p\}$ and

$$
S^{4 n-1} \longrightarrow X \stackrel{a}{\longrightarrow} S^{4 n-1}
$$

is a homotopy equivalence.

By Lemma 3.3 we have $E \circ \alpha \simeq p \circ \gamma$. This together with Lemma 3.4 implies there is a homotopy pullback diagram

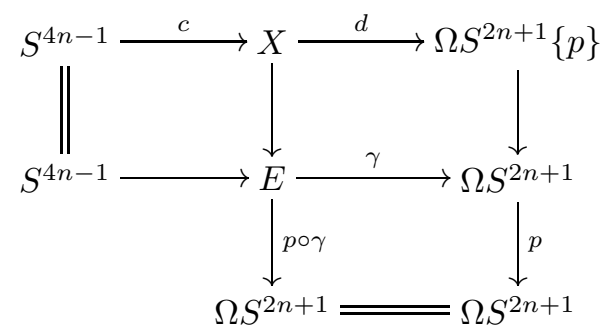

for some maps $c$ and $d$. Lemma 3.4 also tells us that the composition

$$
S^{4 n-1} \stackrel{c}{\longrightarrow} X \longrightarrow E \stackrel{\alpha}{\longrightarrow} S^{2 n}
$$

is homotopic to the Whitehead product $[\iota, \iota]$ plus some perturbation by $p$-torsion. Thus $a \circ c$ is a retraction of $S^{4 n-1}$ off $X$. Hence the fibration

$$
S^{4 n-1} \stackrel{c}{\longrightarrow} X \stackrel{d}{\longrightarrow} \Omega S^{2 n+1}\{p\}
$$

splits and we have a homotopy equivalence

$$
X \stackrel{a \times d}{\longrightarrow} S^{4 n-1} \times \Omega S^{2 n+1}\{p\} .
$$

\section{REFERENCES}

[A] D. Anick, Differential Algebras in Topology, AK Peters, (1993). MR 94h:55020

[CMN] F.R. Cohen, J.C. Moore, and J.A. Neisendorfer, Torsion in homotopy groups, Annals of Math. 109 (1979), 121-168. MR 80e:55024

[G1] B. Gray, On the iterated suspension, Topology 27 (1988), 301-310. MR 89h:55016

[G2] B. Gray, EHP Spectra and Periodicity I: Geometric Constructions, Trans. Amer. Math. Soc. 340 (1993), 595-616. MR 94c:55015

[H] J.R. Harper, A proof of Gray's conjecture, Contemp. Math. 96 (1989), 189-195. MR 91b:55013

[S] P. Selick, Odd primary torsion in $\pi_{k}\left(S^{3}\right)$, Topology 17 (1978), 407-412. MR 80c:55010

[T] S.D. Theriault, Properties of Anick's spaces, Trans. Amer. Math. Soc. 353 (2001), 10091037. MR 2001f:55012

Department of Mathematics, University of Virginia, Charlottesville, Virginia 22904

E-mail address: st7b@virginia.edu

Current address: Department of Mathematical Sciences, University of Aberdeen, Aberdeen, AB24 3UE, United Kingdom

E-mail address: s.theriault@maths.abdn.ac.uk 